\title{
Person-to-Person Cancer Transmission via Allogenic Blood Transfusion
}

\author{
Eugen Molodysky ${ }^{1 *}$, Ross Grant ${ }^{1,2,3}$
}

\begin{abstract}
Despite the recognized capability of Circulating Tumor Cells (CTCs) to seed tumors, allogenic blood transfusions are not presently screened for the presence of CTCs. Previous research has examined blood transfusions and the associated risk of cancer recurrence, but not cancer of unknown primary (CUP) occurrence. The Hypothesis explored in this paper proposes that there is potential for cancers to be transmitted from donor-to-patient via CTCs in either blood transfusions or organ transplants or both. This proposed haematogenic tumor transmission will be discussed in relation to two scenarios involving the introduction of donor-derived CTC's from allogeneic blood transfusions into either known cancer surgery patients or into non-cancer patients. The source of CTCs arises either from the donor with a 'clinically dormant cancer' or a 'pre-clinical cancer' existing as yet undiagnosed, in the donor. Given the significant number of allogenic blood transfusions that occur worldwide on a yearly basis, allogenic blood transfusions have the potential to expose a substantial number of non-cancer recipients to the transmission of CTCs and associated tumor risk. This risk is greatly amplified in the low-income nations where the blood collection and processing protocols, including exclusion and screening criteria are less stringent than those in high-income countries.
\end{abstract}

Keywords: Circulating- tumor- blood- transfusion

Asian Pac J Cancer Prev, 22 (3), 641-649

\section{Introduction}

\section{Cancer Survival}

The lifetime risk of a cancer diagnosis is estimated to be 1 in 2 males and 1 in 3 females in the United States. Cancer-related mortality is approximately 1 in 4 males and 1 in 5 females (National Cancer Institute, 2014). In 2004, a review of randomised clinical trials yielded disappointing results for 5-year survival benefit attributable solely to chemotherapy in adults for 22 major malignancies (National Cancer Institute, 2014). A review by Morgan et al (2004) found the overall contribution of curative and adjuvant chemotherapy to 5 -year in adults to be $2.1 \%$ in the USA. A decade later, in 2014, a review of nine of the most common advanced cancers in the USA showed the median overall survival rates were short, ranging from 4-24 months, despite chemotherapy (Rajagopal et al., 2014).

\section{Clinically Dormant Cancer}

Cancer dormancy refers to the prolonged clinical disease-free time between removal of the primary tumor and recurrence and is known to be a major contributor to morbidity and mortality (Lutz and Heemann, 2003; Yachida et al., 2010).
Crowley and Seigler (1990) analysed 7,104 patients with melanoma and identified 168 who experienced their first recurrence 10 or more years after diagnosis of melanoma, for an incidence of $2.4 \%$. The study also identified 483 patients who had 10 or more years without a subsequent recurrence. That is, of the 651 patients with a long disease-free interval, 25\% (168 of 651) developed recurrent disease. Recurrent malignant melanoma has also been reported after a 35-year disease-free interval (Tahery and Moy, 1993). In breast cancer, 20\% of clinically disease-free patients relapse between 17-25 years after mastectomy and, from 10-20 years, the rate being steady around 1.5\% (Karrison et al., 1999; Saphner et al., 1996; Demicheli et al., 1996).

\section{Pre-clinical Cancer}

Human cancers commonly progress, taking several years before a diagnosis is made, allowing a label such as 'advanced cancer' to mask the true pre-clinical period in which most cancers remain undiagnosed. 'Advanced' typically refers to patients with metastatic disease that cannot be removed or cured (Lutz and Heemann, 2003). For example, Yachinda et al., (2010) have developed a model that forecasts 3 critical times in tumour evolution. They estimate an average of 11.7 years from the initial

${ }^{1}$ Sydney Medical School, University of Sydney, Sydney, Australia. ${ }^{2}$ School of Medical Sciences, University of NSW, Sydney, Australia. ${ }^{3}$ Australasian Research Institute, Sydney Adventist Hospital, Wahroonga, Sydney Australia. *For Correspondence: molodysky@ozemail.com.au 
tumorigenesis until the birth of the cell giving rise to the parental clone, an average of 6.8 years from then until the birth of the cell giving rise to the index lesion, and an average of 2.7 years from then until the patients' death. This is consistent with the observation that $53 \%$ of pancreatic cancer patients currently present with stage IV disease, at which time it is referred to as 'advanced' [with metastases to distant lymph nodes or organs, and where surgical excision is not possible] (Rajagopal et al., 2014). Further, at initial diagnosis, $57 \%$ of patients initially present with 'advanced' or stage IV non-small cell lung cancer, while $20 \%$ of patients initially present with 'advanced' colorectal cancer (Rajagopal et al., 2014). Some cancers are aggressive, with significant variation. Most human cancers double their tumor volume within 60 to 700 days. The fastest growing $5 \%$ of breast cancer can reach $1-2 \mathrm{~cm}$ within a year while the slowest growing $5 \%$ reach this size within 5 decades (Klein, 2013). On average, a $10 \mathrm{~mm}$ breast tumor has a median volume doubling time of 260 days, and takes 20.7 years before reaching 1 billion cells to become screen detectable (Gøtzsche et al., 2012). Conventional guidelines for the early detection of cancer recommend routine screening for individuals aged 45 and above (Smith et al., 2002), yet the pre-clinical stage may well precede this milestone, providing a prolonged period/window where early detection (and intervention) may be possible.

\section{Metastasis without a primary: CUP}

Cancer of Unknown Primary (CUP) comprises a heterogeneous disease group with a diagnosis of metastatic malignancy in the absence of an identifiable primary site after a diagnostic work up, with CUPs accounting for 3-5\% of all malignancies (Massard et al., 2011).

\section{CTCS AS SEEDS OF METASTASIS}

Paget's 1889 conception of the 'seed and soil' hypothesis of cancer metastasis still holds (Fidler, 2003). Circulating Tumour cells (CTCs) were first described in 1869 by an Australian, Dr Thomas Ashworth (Ashworth, 1869) and are now well documented. CTCs are bloodborne cells from either primary or secondary tumors that have migrated into the circulatory system. Over the last three decades, significant research into CTC biological properties has illuminated the critical role these cells play in the metastatic process (Young et al., 2012; O'Flaherty et al., 20102; Polzer et al., 2014). Metastasis is thought to begin with the epithelial-mesenchymal transition (EMT), a cascade of events in which tumor cells lose their 'epithelial' characteristics and become akin to mesenchymal cells with the ability to spread and invade tissue (Polyak and Weinberg, 2009).

Robust evidence exists for the tumorgenicity of CTCs (Strauss and Thomas, 2010; Toloudi et al., 2011; Desai et al., 2012; Hodgkinson et al., 2014). Studies have confirmed that CTCs do in fact reflect the molecular features of cells within primary tumor masses and share the cellular and molecular features of 'cancer-like' stem cells (O’Flaherty et al., 2012; Polzer et al., 2009; Toloudi et al., 2011). CTCs are now accepted as the progenitors for the development of additional tumors (Toloudi et al.,
2011; Gupta and Massagué, 2006), essentially constituting the 'seeds' for metastasis.

With the overwhelming majority of cancer deaths, estimated at $90 \%$, resulting from metastatic spread of the primary tumor to distant lymph nodes or organs (Christofori, 2006), newer diagnostic methods for the early diagnosis of cancer cell burden remains central to preventing metastasis and cancer-related mortality.

\section{CTCs as a Potential 'Liquid Biopsy'for Cancer}

CTC research has recently exploded as new technology platforms enable their capture, confirmation and categorisation (Nagrath et al., 2007, Andergassen et al., 2016). A growing body of evidence now confirms the presence of CTCs in the blood of cancer patients as an important indicator of the potential for metastatic disease, as well as poor prognosis (Nagrath et al., 2007; Allan and Keeney, 2010; Danila et al., 2011; Parkinson et al., 2012). Compared with the well-described difficulties of obtaining tissue biopsies from localized tumor sites, CTC as a 'liquid biopsy', may further provide 'real-time' information of metastasis in action, and hence, the patient's disease status (Parkinson et al., 2012), thus narrowing the timeline for detecting disease progression.

Of particular note is the emerging correlation between the number of CTCs detected and the stages of cancer, where higher CTC counts are associated with the later stages of progression (Nagrath et al., 2007; Allan and Keeney, 2010). Numerous studies have highlighted the importance of CTCs in improving clinical decision making when used in (i) early stage detection of cancer, (ii) predicting the risk of recurrence, (iii) assessing the response to treatment, (iv) selecting patients for adjuvant therapy, and (v) guiding effective treatment selection (Nagrath et al., 2007; Allan and Keeney, 2010; Danila et al., 2011; Parkinson et al., 2012; Krebs et al., 2014; Tognela et al., 2015; Ghossein et al., 1999). As blood tests are safe, cost effective and readily repeatable, information about CTC burden captured from blood samples are likely to provide a high level of clinical utility.

\section{Detecting and Isolating CTCs}

Given the simplicity of routine blood sampling, testing for CTCs may be particularly useful during the pre-clinical period of cancer growth, before the overt symptoms of clinical disease emerge. While the detection of CTCs has substantial prognostic and therapeutic implications, their rarity in the circulation does impose technical challenges (Parkinson et al., 2012; Tognela et al., 2015; Ghossein et al., 1999). CTCs are estimated to be as few as 1-10 CTCs per $\mathrm{mL}$ of circulating whole blood (Tanaka et al., 2009), with even lower numbers in the early stages of cancer. This low frequency translates to isolating 1 cell per 109 hematologic cells in a patient with metastatic disease (Nagrath et al., 2007), rendering further analysis highly challenging.

The challenges of detection and categorisation are further exacerbated by the difficulties associated with cancer cell heterogeneity. The phenotype of CTCs has not been fully defined (Allan and Keeney, 2010; Ghossein et al., 1999). Although CTCs were initially characterised 
as non-leukocytic, nucleated cells that were typically of epithelial origin, we now know that morphological features of CTCs are less well defined and may vary by cancer type, stage and treatment state (Tanaka et al., 2009; Miller et al., 2010). These challenges together coalesce to create the demand for highly sensitive, robust and standardised platforms for the capture and analysis of CTCs. In spite of these challenges, current technologies do provide reliable, though costly and often time consuming, detection of CTC's (Bankó et al., 2019).

Despite the well-established tumorgenicity of CTCs and abundant research interest in its clinical utility, current research has not yet investigated the role of CTCs in donor-derived cancer transmission.

\section{TYPESOF DONOR-DERIVED CANCER TRANSMISSION}

Person-to-person transmission of cancer may occur (1) via organ donation transmission, (2) via cell implantation transmission and (3) via blood donation transmission directly.

1. Transmission by Organ Donation (Donortransmitted events/cancer).

Wimmer et al. were able to show that compared to the normal population, renal transplant patients had a 4.7 times higher risk of developing CUPs (Wimmer et al., 2007).

In 2014, transplants in the USA alone totalled 29,533 (US department of Health and Human Services).

\section{Clinically Dormant Cancer(s)}

Reported cases of allogenic donor-derived tumor transmission date back to the 1960's whereby macroscopically normal renal transplants from a donor who died of metastatic disease resulted in the same carcinoma-related death of both recipients, bronchial carcinoma and epidermoid carcinoma respectively (Martin et al., 1965; McIntosh et al., 1965). As recently as 2015, Yamacake et al. described the cases of 2 kidney transplant recipients who had intestinal carcinoma from the same deceased donor.

In respect of transmission of malignancy by organ donation, Strauss et al., (2010) reported that melanoma cells can remain dormant at distant sites for decades in immune-competent patients, only to reactivate after organ transplantation to an immune-suppressed recipient. That is, the transmission of melanoma by apparent disease-free organ donors.

'Clinical cancer dormancy' is frequently observed in other cancers (Spiliotaki et al., 2014; Aguirre-Ghiso, 2007). Transplant-related malignancies are known to be a major contributor of morbidity and mortality in the organ-recipient population with transplant-related malignancies developing in $15-20 \%$ of graft recipients after 10 years (Crowley and Seigler, 1990).

The Israel Penn International Transplant Registry is among the largest and most comprehensive data base for transplant-related malignancies. Buell et al., (2004) reported as early as 2004, that there were 124 cases of confirmed cancer transmissions from 296 donors with known or incidentally diagnosed malignancies recorded in the registry from 1965 to 2003 .

\section{Pre-clinical Cancers(s)}

The risk of having an organ donor with undetected malignancy ranges between $1.3 \%$ and $2 \%$ (Birkeland and Storm, 2002). The 2013 report of donor-derived transmission events by the Organ Procurement Transplant Network Ad Hoc Disease Transmission Advisory Committee (DTAC) showed an increase of $43 \%$ in the number of potential donor-derived transmission events (PDDTE) reported and reviewed, when compared with 2012. There were 65 donors reported with potential malignancy events of which 5 were classified as 'proven/ probable' transmission, with 8 affected recipients and 2 deaths (Green et al., 2015).

Retrospective analysis of the United Kingdom Transplant Registry for the period 2001 to 2010 (Desai et al., 2012), identified 15 recipients who developed donor-transmitted cancer from 13 donors. None of these donors had a history of past or active cancer at the time of the transplantation. Although all donors underwent assessment to detect transmissible diseases, cross-sectional-imaging and tumor markers were not routinely requested before donation. Desai et al. also notes in this retrospective analysis, that in a number of cases where cancer developed in the recipient. (i) donor origin was neither suspected nor investigated, and (ii) it is possible that a number of recipients may have died with a transmitted cancer that was never identified.

It has been assumed that the recipient malignancy was derived (solely) from the donor organ. However, another source is possible; this perspective postulates that the donor-derived malignancies may have been the result of the transmission of CTCs in the blood contained / entrained in the allogenic donor organ vasculature or the result of transmission of CTCs during an allogenic blood transfusion or both.

\section{2-Transmission by Cell Implantation of Primary Tumor Cells or CTCs}

Patient-derived xenograft (PDX) of Primary Tumor Cells

Several recent comparative studies suggest that patient-derived tumor growth in mice maintain many of the important characteristics of the original tumor (Lum et al., 2012; Peng et al., 2013). Donor-derived xenografting of tissue tumor cells is also known to replicate the metastatic behaviour of clinical cancer (Hiroshima et al., 2015; Decaudin, 2011).

\section{Patient-derived xenograft (PDX) of CTCs}

A significant recent development in cancer research has been the detection, isolation and characterisation of CTCs, with CTCs being used to generate PDX experimental models of cancer.

Blood samples from 6 patients with chemotherapynaïve, extensive-stage small-cell lung cancer (SCLC) were enriched for CTCs and implanted into one or both flanks of immunocompromised mice, resulted in the formation of SCLC tumors (Hodgkinson et al., 2014). Critically, this study was able to demonstrate preservation of morphological and genetic characteristics. Toyoshima 
et al. (2015) showed that tumor-initiating cells are present in the peripheral blood of advanced gastric cancer patients through the establishment of in vivo transplantable tumors in mice from patients CTCs. CTCs have also been used to generate other cancers including breast cancer and prostate cancer PDX models (Baccelli et al., 2013; Yu et al., 2014; Vidal et al., 2015; Williams et al., 2015).

\section{3- Transmission by Blood Donation}

Autologous Blood Transfusions / Intra-operative blood salvage (IBS)

The risk of tumor transmission via blood transfusion has been the subject of past research. While the difference in the risk of cancer recurrence arising from autologous and allogenic blood transfusions has been examined, research has primarily examined the association with the risk of cancer recurrence but not CUP occurrence (Salunkhe et al., 2015; Vamvakas and Blajchman, 2009; Klein, 1995; Li et al., 2015; Waters et al., 2012; Vamvakas, 1995; Gakhar et al., 2013; Heiss et al., 1994). Numerous meta-analyses of intra-operative blood salvage (IBS) in cancer surgery exist (Li et al., 2015; Waters et al., 2012; Vamvakas, 1995; Zhai and Sun, 2013). However, these focus on primary site cancer cells shed during the surgical procedure, which are distinct from blood-borne CTCs. Of note, to date, no study has been conducted to examine the role of donor-derived CTCs in facilitating a CUP in the recipient, following an allogenic blood transfusion.

\section{Allogenic Blood Transfusions}

Intraoperative blood salvage (IBS) is a technique frequently employed in major blood loss surgery, however it has been restricted in cancer surgery patients because of contaminating cancer cells 'shed' during the surgery and their systemic dissemination following reinfusion.

For the majority of patients requiring blood transfusion, the source is allogenic, that is, donor-derived (Salunkhe et al., 2015). It is estimated that each year 118.5 million blood donations are collected globally (World Health Organization, 2020).

Since the end of the Second World War, blood banks have inclined towards fractioning whole blood into components (red blood cell, plasma and platelets) for economic efficiency and logistical longevity (Murdock et al., 2014). Leukocyte reduction via filtration is less effective on whole blood compared to pre-separated blood components (Bruil et al., 1995; Singh and Kumar, 2009), rendering whole blood transfusion recipients at a relatively higher risk of encountering CTCs. According to the World Health Organisation, whole blood $60 \%$ of the total number of transfusions, defined as blood 'transfused to a patient in an unmodified state' occur in low or middleincome countries, countries (World Health Organization, 2020). This means lower to middle income countries are disproportionately placed at risk of receiving CTCs via blood that is not leuko-reduced.

Further, whole blood transfusion is still routinely used in military circumstances and austere environments where a well-equipped blood bank is not accessible (Auten et al., 2015; Beckett et al., 2015). In addition, whole blood transfusion has been found to possess superior therapeutic benefits over blood components in paediatric cardiac surgery and shock resuscitation (Murdock et al., 2014; Jobes et al., 2015; Rhee et al., 2015), which merit its continued usage.

The Australian Red Cross Blood Service (ARCBS) tests for five transfusion transmissible diseases (HIV/ AIDS, Hepatitis B, Hepatitis C, human T-cell lymphoproliferative virus [HTLV] and syphilis). The ARCBS excludes donors with a history of cancers such as leukaemia, lymphoma and myeloma, however ARBCS does accept people who remain free of cancer for five

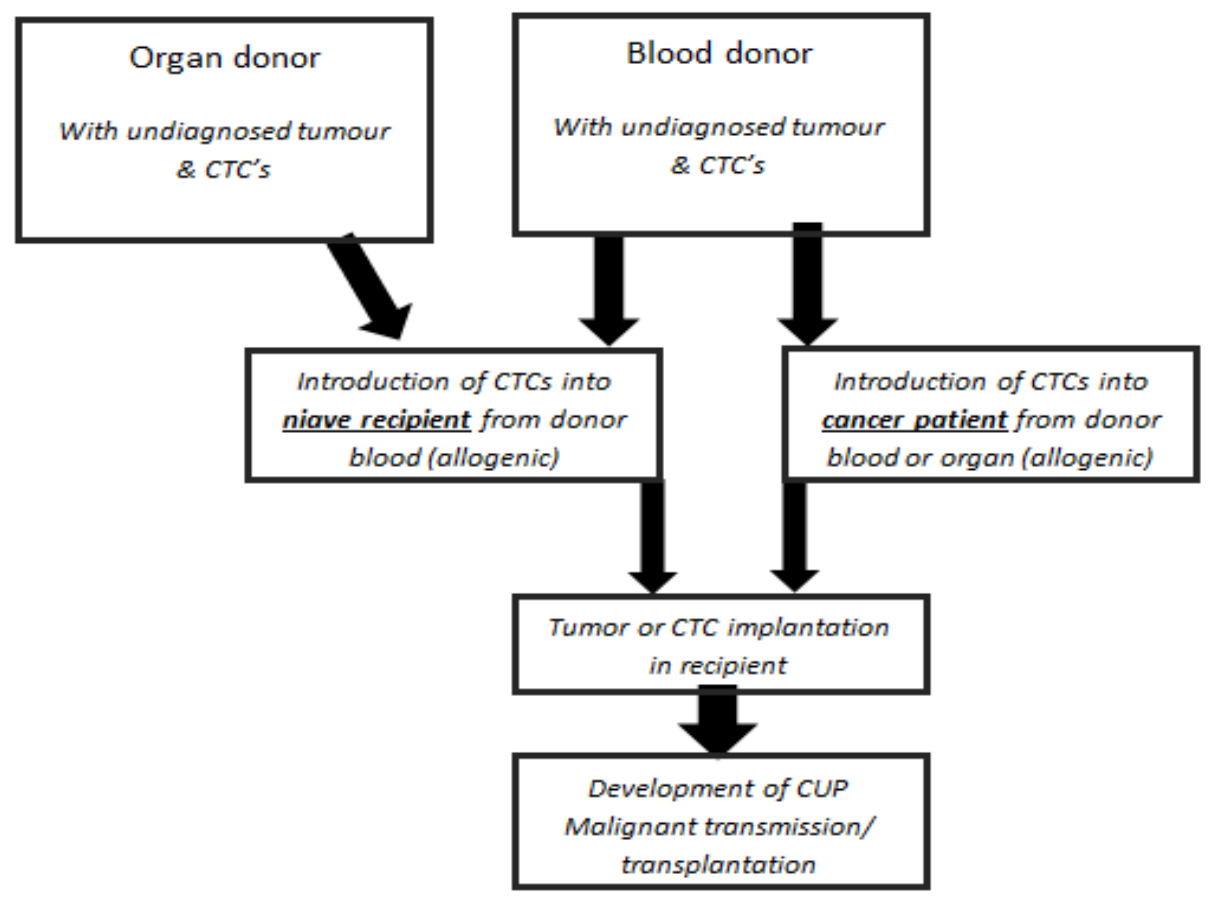

Figure 1. Haematogenic Cancer Transmission Scenarios. 
years after the completion of their treatment. In contrast, the American Red Cross Blood Service excludes donors of cancers of the blood, but does accept donations from patients with solid cancers after 12 months have passed since successful treatment (Catling et al., 2008). Current recommendations set by $\mathrm{WHO}$ for safe screening of donor blood products state that, "For individuals with a past history of solid malignant tumour, BTS (blood transfusion services) may consider acceptance if 5 years or more since completion of successful curative treatment (WHO, 2012).

This Perspective postulates that the current practices for allogenic blood transfusions may place recipients at risk of tumor transmission. This stems primarily from two factors:

Firstly, the current blood donor selection criteria includes, not excludes, individuals who remain diseasefree of cancer for one years after the completion of their cancer treatment in the USA and 5 years in Australia and those countries adopting the WHO recommendations (Australian Red Cross Blood Service; Catling et al., 2008, WHO 2012). As 'clinical dormancy' represents a reservoir from 10 to 30 years (Strauss and Thomas, 2010; Spiliotaki et al., 2014), the current selection criteria may allow relapsing cancer patients to donate blood. Further the failure to diagnose cancer in its pre-clinical phase, especially as evidenced and represented by the plethora of 'advanced cancer' diagnoses, may again allow for preclinical cancer patients to also donate blood (and organs). This situation is most likely to be compounded in the lower-income nations.

Secondly, current filtration practices for leukocyte reduction remove only $80 \%$ to $99 \%$ of leukocytes (Singh and Kumar, 2009). Previous experiments into leukocyte filtration show inconsistent results for complete removal of primary site tumor cells shed during surgery (Bruil et al., 1995; Catling et al., 2008; Edelman et al., 1996). As the use of leukocyte reduction filters are unable to guarantee complete elimination of contaminating cancer cells shed during surgery from the reinfused blood (Hansen et al., 1993; Hansen et al., 1997), it is likely that the standard leukocyte reduction filters used by blood banks are also unable to guarantee complete elimination of CTCs. In particular, previous evidence used to purport the effective removal of tumor cells in blood after leukocyte reduction, has been criticised as flawed. Criticisms range from inferior detection sensitivity, to overestimating tumor cell reduction, to mistakenly generalising tumor cell filtration in plasma as identical with filtration in blood (Hansen, 2006).

The likelihood of incomplete CTC elimination is increased by the fact that CTCs are heterogeneous, yet not dissimilar in size to leukocytes (Allan and Keeney, 2010) with the overlap in size favouring the smaller CTCs. Whole blood donations are not currently screened for CTCs.

The risk of recipients developing cancer from donor-derived CTCs may depend on both the number and characteristics of the CTCs, as well as the vulnerability of the recipient's immune system that places the recipient at additional risk.

While irradiation of allogenic blood is typically reserved for the immune-compromised, its use has been demonstrated to provide the efficient elimination of shed cancer cells contaminating the blood salvaged for autologous blood transfusion, following irradiation with 50 Gy (Hansen et al., 1999). While CTCs from donors in cancer 'remission' or from donors with pre-clinical cancer represent a real risk for all recipients who are immune compromised a degree of risk may also extend to otherwise healthy individuals who exhibit a degree of immune incompetence such as reduced natural killer (NK) cell function. NK cells are particularly important in recognising and destroying cancer cells including CTC's and reduced NK activity has been shown to increase the risk of tumor development (Wu and Lanier, 2003; Moon and Powis, 2019; Vidal et al., 2019). Importantly a significant reduction in NK activity has been linked to multiple lifestyle factors including, obesity (O'Shea and Hogan, 2019), psychosocial stress (Vitaliano et al., 1998), reduced sleep (Shakhar et al., 2007), and type 2 diabetes (Shakhar et al., 2007), suggesting that many within even the general population are compromised in their ability to eliminate CTC's, and are therefore at increased risk of tumor development.

\section{HYPOTHESIS}

Past research has focused on reintroduction/reinfusion of the patient-derived primary site tumor cells shed during surgery via autologous blood transfusions (Waters et al., 2012; Klein, 1995; Raghavan and Marik, 2005). That is, non-donor-derived primary site tumor cells which are 'salvaged' from the operating site and are 'salvage' and are reinfused into the patient, potentially facilitating metastasis of the original malignancy.

It is (thus) hypothesised, that the apparent contradictory results for occurrence of malignancies associated with peri-operative blood transfusions (Salunkhe et al., 2015; Vamvakas and Blajchman, 2009; Klein, 1995; Li et al., 2015; Waters et al., 2012; Vamvakas, 1995; Gakhar et al., 2013; Heiss et al., 1994), or occurrence of malignancies of unknown primary origin, may be elucidated further by proposing two additional distinct scenarios of haematogenic tumor transmission (Figure 1), namely:

1- Introduction of donor-derived CTCs from allogenic blood transfusions into known cancer surgery patients. That is, the donor has not yet presented with a sign or symptom of (i) recurrence (5yrs) (clinically dormant cancer) or (ii) first time cancer (pre-clinical cancer). Thus this may account for the presence/occurrence of a new malignancy/cancer of unknown primary origin (CUP) in the recipient.

2- Introduction of donor-derived CTCs during allogenic blood transfusions into niave/non-cancer patients. Neither individual (donor or recipient) having yet presented with signs or symptoms of cancer (clinical disease). Thus this may account for the occurrence of a new malignancy/cancer of unknown primary origin (CUP) in the recipient.

Therefore, person-to-person transmission of cancer may arise via organ donation transmission of CTCs entrained in the organ vasculature or via allogenic blood donation transmission of CTCs directly at the time of 
transplantation surgery, together or independently.

\section{CONCLUDING REMARKS}

The evidence strongly supports tumor transmission from the allogenic organ donor during transplantation. It has been assumed that the recipient malignancy described in the literature was derived (solely) from the donor organ. However, we postulate that the donor-derived malignancies may have actually been derived from transmission of CTCs present in the blood that was either entrained in the donor organ vasculature or provided as an allogenic blood transfusion during the transplantation surgery.

While cancer transmission through tissue tumor cells is well accepted, to date, no study has yet explored the role of donor-derived CTCs in facilitating cancer (CUP) genesis in the recipient following either an organ donation or an allogenic blood donor transfusion or both.

The capacity of donated CTCs to proliferate in the recipient's blood may not be dissimilar to donor leukocyte proliferative activity in GVHD, especially in those with reduced immune function (e.g. reduced NK cell activity).

The risks of malignancy/cancer transmission from donors with clinically dormant cancers and preclinical cancers during organ donation or allogenic blood transfusions require urgent and immediate reassurance initiatives/measures. This article, in providing a perspective, focuses primarily on high-income nations and in turn heralding the even greater need for world-wide attention to the other members of our global family - the low-income nations.

\section{Recommendations for consideration}

1. Donor clinical research to include (i) retrospective secondary analysis of 5-10 years blood transfusion data to assess relative cancer risk in recipients and (ii) prospective studies to create a robust and comprehensive set of metadata detailing donor and recipient details and relative cancer risk to improve our understanding and enable stringent hypothesis testing.

2. Donor exclusion criteria to be expanded to exclude any person with a history of cancer, whether or not they have been cancer free for 5 years (clinically dormant cancer patients).

3. Donor exclusion criteria to be expanded to exclude any person who falls into the 'at risk' group for pre-clinical cancer, that is, above the age of 45 , who begin to cross over into the various age-relate national (USA) cancer screening thresholds.

4. Donor allogenic blood screening platforms be explored that include the capture, confirmation and categorisation of CTCs (pre-clinical cancer patients) that may complement existing allogenic blood transfusion protocols.

It is noted that most current methodologies for detecting CTC's are labor intensive and expensive, limiting the use of this important marker in lower income countries. However, technology in this field is rapidly expanding (Hendricks et al., 2020) and cost effective options are anticipated to be available in the not too distant future.
5. Review blood leuko-reduction and irradiation protocols.

In context of the lengthy latency period of most human cancers before diagnosis, and the preponderance of 'advanced cancer' diagnoses, the likelihood of CTCs presenting in blood during this window period represents a unique opportunity to identify pre-clinical cancer in its early stages. Aside from also protecting organ and blood recipients from the risk of cancer transmission from clinically dormant cancer(s), with the emergence of CTC screening platforms on the horizon, these additional safety measure(s) will shift the focus to early detection and management of cancer in the general population.

\section{Author Contribution Statement}

Conceptualisation; EM, Writing original draft manuscript; EM. Data/reference curation EM,RG. Administration RG. Preparation of revised manuscript; $\mathrm{RG}$, Review \& submission of final draft of manuscript, EM, RG.

\section{Acknowledgments}

\section{Ethical statement}

This article does not contain any studies involving human (or animal) participants performed by any of the authors

\section{Conflict of interest}

The authors have no conflict of interest.

\section{References}

Aguirre-Ghiso JA (2007). Models, mechanisms and clinical evidence for cancer dormancy. Nat Rev Cancer, 7, 834-46.

Allan AL, Keeney M (2010). Circulating tumor cell analysis: Technical and statistical considerations for application to the clinic. J Oncol, 2010, 426218.

Andergassen U, Kölbl AC, Mahner S, Jeschke U (2016). Real-time RT-PCR systems for CTC detection from blood samples of breast cancer and gynaecological tumour patients (Review). Oncol Rep, 35, 1905-15.

Ashworth TR (1869). A case of cancer in which cells similar to those in the tumors were seen in the blood after death. $A M J, 14,146-7$.

Australian Red Cross Blood Service. Blood Testing and Safety. http://www.donateblood.com.au/eligibility/blood-testingand-safety. Accessed 12 June 2019.

Australian Red Cross Blood Service. I need to know about irradiation. Transfusion Fact Sheet Volume 1, Number 8. http://mytransfusion.com.au/sites/default/files/I_need_to_ know_about_irradiation.pdf. Accessed 12 June 2019.

Auten JD, Lunceford NL, Horton JL, et al (2015). The safety of early fresh, whole blood transfusion among severely battle injured at US Marine Corps forward surgical care facilities in Afghanistan. J Trauma Acute Care Surg, 79, 790-6.

Baccelli I, Schneeweiss A, Riethdorf S, et al (2013). Identification of a population of blood circulating tumor cells from breast cancer patients that initiates metastasis in a xenograft assay. Nat Biotechnol, 31, 539-44.

Bankó P, Lee SY, Nagygyörgy V, et al (2019). Technologies for circulating tumor cell separation from whole blood. 
J Hematol Oncol, 12, 48.

Beckett A, Callum J, da Luz LT, et al (2015). Fresh whole blood transfusion capability for Special Operations Forces. Can J Surg, 58, S153-6.

Birkeland SA, Storm HH (2002). Risk for tumor and other disease transmission by transplantation: A population-based study of unrecognized malignancies and other diseases in organ donors. Transplantation, 74, 1409-13.

Bruil A, Beugeling T, Feijen J, van Aken WG (1995). The mechanisms of leukocyte removal by filtration. Transfus Med Rev, 9, 145-66.

Buell JF, Beebe TM, Trofe J, et al (2004). Donor transmitted malignancies. Ann Transpl, 9, 53-6.

Catling S, Williams S, Freites O, et al (2008). Use of a leucocyte filter to remove tumour cells from intra-operative cell salvage blood. Anaesthesia, 63, 1332-8.

Christofori G (2006). New signals from Invasive front. Nature, 441, 444-50.

Crowley NJ, Seigler HF (1990). Late recurrence of malignant melanoma: Analysis of 168 patients. Ann Surg, 212, 173-7.

Danila DC, Fleisher M, Scher HI (2011). Circulating tumor cells as biomarkers in prostate cancer. Clin Cancer Res, 17, 3903-12.

Decaudin D (2011). Primary human tumor xenografted models ('tumorgrafts') for good management of patients with cancer. Anti-Cancer Drug, 22, 827-41.

Demicheli R, Abbattista A, Miceli R, Valagussa P, Bonadonna $\mathrm{G}$ (1996). Time distribution of the recurrence risk for breast cancer patients undergoing mastectomy: Further support about the concept of tumor dormancy. Breast Cancer Res Tr, 41, 177-85.

Desai R, Collett D, Watson CJ, et al (2012). Cancer transmission from organ donors-unavoidable but low risk. Transplantation, 94, 1200-7.

Edelman MJ, Potter P, Mahaffey KG, Frink R, Leidich RB (1996). The potential for reintroduction of tumor cells during intraoperative blood salvage: Reduction of risk with use of the RC-400 leukocyte depletion filter. Urology, 47, 179-81.

Fidler IJ (2003). The pathogenesis of cancer metastasis: the 'seed and soil' hypothesis revisited. Nat Rev Cancer, 3, 453-8.

Gakhar H, Bagouri M, Bommireddy R, Klezl Z (2013). Role of intraoperative red cell salvage and autologus transfusion in metastatic spine surgery: a pilot study and review of literature. Asian Spine J, 7, 167-72.

Ghossein RA, Bhattacharya S, Rosai J (1999). Molecular detection of micrometastases and circulating tumor cells in solid tumors. Clin Cancer Res, 5, 1950-60.

Gøtzsche PC, Jørgensen KJ, Zahl PH, Mæhlen J (2012). Why mammography screening has not lived up to expectations from the randomised trials. Cancer Causes Control, 23, $15-21$.

Green M, Covington S, Taranto S, et al (2015). Donor-derived transmission events in 2013: A report of the organ procurement transplant network Ad Hoc disease transmission advisory committee. Transplantation, 99, 282-7.

Gupta GP, Massagué J (2006). Cancer metastasis: Building a framework. Cell, 126, 679-95.

Hansen E (2006). Failed evidence of tumour cell removal from salvaged blood after leucocyte depletion. Transfusion Med, 16, 213-4.

Hansen E FI, Knuechel-Clarke R, et al (1993). Tumor cells in intraoperatively salvaged blood and their elimination by leukocyte filters or gamma-irradiation (abstract). Anaesthesiology, 79, A122.

Hansen E, Altmeppen J, Taiger K (1997). Autologous haemotherapy in malignant diseases. Baillieres Clin Anaesthesiol, 11, 335-50.
Hansen E, Knuechel R, Altmeppen J, Taeger K (1999). Blood irradiation for intraoperative autotransfusion in cancer surgery: Demonstration of efficient elimination of contaminating tumor cells. Transfusion, 39, 608-15.

Heiss MM, Mempel W, Delanoff C, et al (1994). Blood transfusion-modulated tumor recurrence: First results of a randomized study of autologous versus allogeneic blood transfusion in colorectal cancer surgery. J Clin Oncol, 12, 1859-67.

Hendricks A, Brandt B, Geisen R, et al. (2020). Isolation and enumeration of CTC in colorectal cancer patients: Introduction of a Novel Cell Imaging Approach and Comparison to Cellular and Molecular Detection Techniques. Cancers (Basel), 12, 2643.

Hiroshima Y, Maawy AA, Katz MHG, et al (2015). Selective efficacy of zoledronic acid on metastasis in a patient-derived orthotopic xenograph (PDOX) nude-mouse model of human pancreatic cancer. J Surg Oncol, 111, 311-5.

Hodgkinson CL, Morrow CJ, Li Y, et al (2014). Tumorigenicity and genetic profiling of circulating tumor cells in small-cell lung cancer. Nat Med, 20, 897-903.

Jobes DR, Sesok-Pizzini D, Friedman D (2015). Reduced transfusion requirement with use of fresh whole blood in pediatric cardiac surgical procedures. Ann Thorac Surg, 99, 1706-11.

Karrison TG, Ferguson DJ, Meier P (1999). Dormancy of mammary carcinoma after mastectomy. J Natl Cancer Inst, 91, 80-5.

Kim JH, Park K, Lee SB, et al (2019). Relationship between natural killer cell activity and glucose control in patients with type 2 diabetes and prediabetes. J Diabetes Investig, 10, 1223-8.

Klein CA (2013). Selection and adaptation during metastatic cancer progression. Nature, 501, 365-72.

Klein HG (1995). Allogeneic transfusion risks in the surgical patient. Am J Surg, 170, 21s-6s.

Krebs MG, Metcalf RL, Carter L, et al (2014). Molecular analysis of circulating tumour cells - Biology and biomarkers. Nat Rev Clin Oncol, 11, 129-44.

Li L, Zhu D, Chen X, et al (2015). Perioperative allogenenic blood transfusion is associated with worse clinical outcome for patients undergoing gastric carcinoma surgery: A metaanalysis. Medicine, 94, e1574.

Lum DH, Matsen C, Welm AL, Welm BE (2012). Overview of human primary tumorgraft models: comparisons with traditional oncology preclinical models and the clinical relevance and utility of primary tumorgrafts in basic and translational oncology research. Curr Protoc Pharmacol, Chapter, 14, Unit 14.22.

Lutz J, Heemann U (2003). Tumours after kidney transplantation. Curr Opin Urol, 13, 105-9.

Martin DC, Rubini M, Rosen VJ (1965). Cadaveric renal homotransplantation with inadvertent transplantation of carcinoma. JAMA, 192, 752-4.

Massard C, Loriot Y, Fizazi K (2011). Carcinomas of an unknown primary origin-diagnosis and treatment. Nat Rev Clin Oncol, 8, 701-10.

McIntosh DA, McPhaul JJ, Peterson EW, et al (1965). Homotransplantation of a cadaver neoplasm and a renal homograft. JAMA, 192, 1171-3.

Miller MC, Doyle GV, Terstappen LW (2010). Significance of circulating tumor cells detected by the Cellsearch System in patients with metastatic breast colorectal and prostate cancer. J Oncol, 2010, 617421.

Moon WY, Powis SJ (2019). Does natural killer cell deficiency (NKD) increase the risk of cancer? NKD may increase the risk of some virus induced cancer. Front. 
Morgan GW, Ward R, Barton M (2004). The contribution of cytotoxic chemotherapy to 5-year survival in adult malignancies. Clin Oncol, 16, 549-60.

Murdock AD, Berséus O, Hervig T, Strandenes G, Lunde TH (2014). Whole blood: The future of traumatic hemorrhagic shock resuscitation. Shock, 41, 62-9.

Nagrath S, Sequist LV, Maheswaran S, et al (2007). Isolation of rare circulating tumour cells in cancer patients by microchip technology. Nature, 450, 1235-9.

National Cancer Institute (2014). Lifetime Risk (Percent) of Dying from Cancer by Site and Race/Ethnicity: Males TU, 2009-2011 (Table 1.19) and Females, Total US, 20092011 (Table 1.20). http://seer.cancer.gov/csr/1975_2011/ results_merged/topic_lifetime_risk_death.pdf, 20 Accessed February 2016.

O'Shea D, Hogan AE (2019). Dysregulation of natural killer cells in obesity. Cancers, 11, 573.

O'Flaherty JD, Gray S, Richard D, et al (2012). Circulating tumour cells, their role in metastasis and their clinical utility in lung cancer. Lung Cancer, 76, 19-25.

Parkinson DR, Dracopoli N, Petty BG, et al (2012). Considerations in the development of circulating tumor cell technology for clinical use. J Transl Med, 10, 138.

Peng S, Creighton CJ, Zhang Y, et al (2013). Tumor grafts derived from patients with head and neck squamous carcinoma authentically maintain the molecular and histologic characteristics of human cancers. $J$ Transl Med, 11, 198.

Polyak K, Weinberg RA (2009). Transitions between epithelial and mesenchymal states: Acquisition of malignant and stem cell traits. Nat Rev Cancer, 9, 265-73.

Polzer B, Medoro G, Pasch S, et al (2014). Molecular profiling of single circulating tumor cells with diagnostic intention. EMBO Mol Med, 6, 1371-86.

Raghavan M, Marik PE (2005). Anemia, allogenic blood transfusion, and immunomodulation in the critically ill. Chest, 127, 295-307.

Rajagopal PS, Nipp RD, Selvaggi KJ (2014). Chemotherapy for advanced cancers. Ann Palliat Med, 3, 203-28.

Rhee P, Inaba K, Pandit V, et al (2015). Early autologous fresh whole blood transfusion leads to less allogeneic transfusions and is safe. $J$ Trauma Acute Care Surg, 78, 729-34.

Salunkhe V, van der Meer PF, de Korte D, Seghatchian J, Gutiérrez L (2015). Development of blood transfusion product pathogen reduction treatments: A review of methods, current applications and demands. Transfus Apher Sci, 52, 19-34.

Saphner T, Tormey DC, Gray R (1996). Annual hazard rates of recurrence for breast cancer after primary therapy. J Clin Oncol, 14, 2738-46.

Shakhar K, Valdimarsdottir HB, Guevarra JS, Bovbjerg DH (2007). Sleep, fatigue, and NK cell activity in healthy volunteers: Significant relationships revealed by within subject analyses. Brain Behav Immun, 21, 180-4.

Singh S, Kumar A (2009). Leukocyte depletion for safe blood transfusion. L Biotechnol, 4, 1140-51.

Smith RA, Cokkinides V, von Eschenbach AC, et al (2002). American Cancer Society guidelines for the early detection of cancer. Cancer J Clin, 52, 8-22.

Spiliotaki M, Mavroudis D, Kapranou K, et al (2014). Evaluation of proliferation and apoptosis markers in circulating tumor cells of women with early breast cancer who are candidates for tumor dormancy. Breast Cancer Res, 16, 485.

Strauss DC, Thomas JM (2010). Transmission of donor melanoma by organ transplantation. Lancet Oncol, 11, 790-6.

Tahery DP, Moy RL (1993). Recurrent malignant melanoma following a 35-year disease-free interval. J Dermatol Surg
Oncol, 19, 161-3.

Tanaka F, Yoneda K, Kondo N, et al (2009). Circulating tumor cell as a diagnostic marker in primary lung cancer. Clin Cancer Res, 15, 6980-6.

Tognela A, Spring KJ, Becker T, et al (2015). Predictive and prognostic value of circulating tumor cell detection in lung cancer: A clinician's perspective. Crit Rev Oncol Hematol, 93, 90-102.

Toloudi M, Apostolou P, Chatziioannou M, Papasotiriou I (2011). Correlation between cancer stem cells and circulating tumor cells and their value. Case Rep Oncol, 4, 44-54.

Toyoshima K, Hayashi A, Kashiwagi M, et al (2015). Analysis of circulating tumor cells derived from advanced gastric cancer. Int J Cancer, 137, 991-8.

US department of Health and Human Services. Organ Procurement and Transplantation Network TitUbS. https:// optn.transplant.hrsa.gov/converge/latestData/rptData.asp. Accessed 12 June 2019.

Vamvakas EC (1995). Perioperative blood transfusion and cancer recurrence: meta-analysis for explanation. Transfusion, $\mathbf{3 5}$, 760-8.

Vamvakas EC, Blajchman MA (2009). Transfusion-related mortality: the ongoing risks of allogeneic blood transfusion and the available strategies for their prevention. Blood, 113, 3406-17.

Vidal AC, Howard LE, Wiggins E, et al (2019). Natural killer cell activity and prostate cancer risk in veteran men undergoing prostate biopsy. Cancer Epidemiol, 62, 101578.

Vidal S, Rodriguez-Bravo V, Quinn S, et al (2015). A targetable GATA2-IGF2 axis confers aggressiveness in lethal prostate cancer. Cancer Cell, 27, 223-39.

Vitaliano PP, Scanlan JM, Ochs HD, et al (1998). Psychosocial stress moderates the relationship of cancer history with natural killer cell activity. Ann Behav Med, 20, 199-208.

Waters JH, Yazer M, Chen YF, Kloke J (2012). Blood salvage and cancer surgery: A meta-analysis of available studies. Transfusion, 52, 2167-73.

Williams ES, Rodriquez-Bravo V, Chippada-Venkata U, et al (2015). Generation of prostate cancer patient derived xenograft models from circulating tumor cells. $J$ Vis Exp, 2015, 3182 .

Wimmer CD, Rentsch M, Crispin A, et al (2007). The Janus face of immunosuppression - De novo malignancy after renal transplantation: The experience of the Transplantation Center Munich. Kidney Int, 71, 1271-8.

World Health Organization (2020). Blood Safety and Availability Fact Sheet 2020. https://www.who.int/news-room/factsheets/detail/blood-safety-and-availability Accessed 11 February 2021.

World Health Organisation (2012) Guidelines on Assessing Donor Suitability for Blood Donation. P59. https:// www.who.int/bloodsafety/publications/BDSelection WHOGuideAssessingDonorSuitability4BloodDonation.pdf. Accessed 11 February 2021.

Wu J, Lanier LL (2003). Natural killer cells and cancer. $A d v$ Cancer Res, 90, 127-56.

Yachida S, Jones S, Bozic I, et al (2010). Distant metastasis occurs late during the genetic evolution of pancreatic cancer. Nature, 467, 1114-7.

Yamaçake KGR, Antonopoulos IM, Piovesan AC, et al (2015). Donor transmission intestinal carcinoma after kidney transplantation: Case report. Transplant Proc, 47, 827-30.

Young R, Pailler E, Billiot F, et al (2012). Circulating tumor cells in lung cancer. Acta Cytologica, 56, 655-60.

Yu M, Bardia A, Aceto N, et al (2014). Ex vivo culture of circulating breast tumor cells for individualized testing of drug susceptibility. Science, 345, 216-20. 
Zhai B, Sun XY (2013). Controversy over the use of intraoperative blood salvage autotransfusion during liver transplantation for hepatocellular carcinoma patients. World J Gastroenterol, 19, 3371-4.

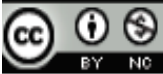

This work is licensed under a Creative Commons AttributionNon Commercial 4.0 International License. 